\title{
Síndrome de desgaste profesional (burnout) en médicos cirujanos especialistas: prevalencia y factores de riesgo
}

\author{
Professional burnout syndrome in specialist surgeons: prevalence and risk factors
}

\author{
Enrique Castañeda-Aguilera* y Javier E. García de-Alba-García \\ Unidad de Investigación Social, Epidemiológica y de Servicios de Salud, Instituto Mexicano del Seguro Social, Guadalajara, Jalisco, México
}

\begin{abstract}
Resumen
Antecedentes: Los médicos cirujanos especialistas están expuestos a factores de riesgo, siendo los más frecuentes los de tipo psicosocial, incluyendo el síndrome de desgaste profesional (burnout) por el tipo de exposición y la diversificación de sus actividades como miembros del equipo de salud, y las repercusiones jurídicas y sociolaborales. Objetivo: Determinar la prevalencia y los factores de riesgo del burnout en médicos cirujanos especialistas. Método: Estudio observacional, descriptivo y transversal, en 296 especialistas. Los datos se procesaron descriptivamente y de manera inferencial con apoyo de los programas SPSS 15.0 y Epi-infoV6.1. Resultados: Hubo respuesta en el 92.5\% de los entrevistados y el burnout se encontró en el 40.2\%. Se detectaron diferencias significativas en edad menor de 40 años, no tener pareja estable o menos de 15 años con pareja, ser médico oncólogo quirúrgico, y tener menos de 10 años de antigüedad profesional o en puesto de trabajo. Conclusiones: El burnout es frecuente (40.2\%) y como factores de riesgo están ser mujer, ser menor de 40 años, no tener pareja estable o menos de 15 años con pareja y que esta no trabaje, no tener hijos, ser médico oncólogo quirúrgico, tener menos de 10 años de antigüedad profesional o en puesto de trabajo, trabajar en turno nocturno, tener contratación definitiva, no tener otro trabajo y trabajar más de 4 horas en él. La afectación de las subescalas se comporta como el síndrome. Hubo correlación negativa con el burnout entre agotamiento emocional y despersonalización, y positiva con falta de realización personal en el trabajo.
\end{abstract}

Palabras Clave: Médicos cirujanos especialistas. Burnout. Agotamiento emocional. Despersonalización. Falta de realización personal.

\begin{abstract}
Background: Medical surgeons specialists are exposed to risk factors, the most frequent being those of the psychosocial type, where burnout syndrome is included due to the type of exposure and diversification of their activities as a member of the health team and the legal and socio-labor repercussions. Objective: To determine the prevalence and risk factors of burnout in medical surgeons. Method: Observational, descriptive and cross-sectional study in 296 specialists. The data was processed descriptively and inferentially with the support of the SPSS 15.0 and Epi-infoV6.1 program. Results: There was a response in $92.5 \%$ of the interviewees and the burnout was found in $40.2 \%$. Significant differences were detected in age under 40 years, not having a stable partner, and < 15 years with your partner, being a medical oncologist, having
\end{abstract}

\section{Correspondencia:}

*Enrique Castañeda-Aguilera

Belisario Domínguez, 1000

Col. Independencia

Fecha de recepción: 15-08-2019

Cir Cir. 2020;88(3):354-360

C.P. 44340, Guadalajara, Jal., México

Fecha de aceptación:20-09-2019

E-mail: ecastaneda03@ hotmail.com

DOI: $10.24875 / C I R U .19001502$

Contents available at PubMed

www.cirugiaycirujanos.com

0009-7411/@ 2019 Academia Mexicana de Cirugía. Publicado por Permanyer. Este es un artículo open access bajo la licencia CC BY-NC-ND (http://creativecommons.org/licenses/by-nc-nd/4.0/). 
$<10$ years of professional seniority and in the workplace. Conclusions: Burnout is frequent (40.2\%), as risk factors are, being: woman; under 40 years old; not having a stable partner, under 15 years with her and not working this, without children; surgical medical oncologist; < 10 years of professional seniority and job position, night shift; definitive hiring; not having another job and more than $4 \mathrm{~h}$ in it. The involvement of the subscales behaves like the syndrome. There was a negative correlation with burnout between emotional exhaustion and depersonalization, and positive with lack of personal fulfillment at work.

Key Words: Medical surgeons specialists. Burnout. Emotional exhaustion. Depersonalization. Lack of personal fulfillment.

\section{Introducción}

El área quirúrgica combina el tratamiento médico clínico con un abordaje intervencionista, donde el paciente recibe asistencia perioperatoria, con actividades de preparación preoperatoria, manejos intraoperatorios y cuidados posoperatorios. El quirófano es un área donde el personal quirúrgico presenta dificultad para el desempeño laboral, por la complejidad de las tareas que se realizan. La bioseguridad y la prevención de riesgos laborales a los que se está expuesto se convierten en un problema para el equipo de salud que desempeña su función en la sala de operaciones, además de estar en contacto con enfermedades como la infección por el virus de la inmunodeficiencia humana, la tuberculosis y la hepatitis, entre otras, a sustancias neurotóxicas, mutagénicas, carcinogénicas y anestésicas, y también ruido, radiaciones ionizantes y no ionizantes, e iluminación, junto con un tiempo de trabajo prolongado, espacios reducidos, posturas y bipedestación prolongada, etc.

El equipo quirúrgico puede estresarse por la conducta de otros miembros, así como por las reacciones inesperadas que puede presentar el paciente durante la cirugía o por los instrumentos que se utilizan durante esta. La desilusión constante y los conflictos internos crean un ambiente complicado y que genera situaciones estresantes que pueden alterar al grupo.

El personal médico quirúrgico está expuesto a factores de riesgo, siendo los más frecuentes los de tipo psicosocial por la diversificación de sus actividades laborales atendiendo a pacientes de consulta externa, hospitalizados, interconsultas en urgencias e intervenciones quirúrgicas que realizan, y tener a su cargo al personal médico becario de pregrado y posgrado, además de trámites administrativos inherentes a los procedimientos médicos que se realizan en el hospital y de asesoría en su respectiva especialidad; sin olvidar la responsabilidad ética profesional y los posibles conflictos de tipo médico-legal a los que están expuestos con el ejercicio de su trabajo.
Los riesgos psicosociales son un fenómeno relevante y han alcanzado rápidamente una considerable notoriedad en el contexto laboral: es el síndrome de desgaste profesional (SDP) o burnout, que clínicamente fue descrito por Freudenberger en $1974^{1}$ como «un estado de fatiga o frustración que se produce por la dedicación a una causa, forma de vida o relación que no produce el esperado refuerzo» de ayuda. En 1977, la Asociación Americana de Psicólogos lo describió en personal que trabaja en servicios humanos, profesionales de salud y educación bajo condiciones difíciles en contacto con usuarios. En 1981, Maslach, et al. $^{2}$ lo estudiaron desde una perspectiva tridimensional: agotamiento emocional (AE), manifestado por pérdida progresiva de energía, cansancio y fatiga; despersonalización (DP), identificada por un cambio negativo de actitudes y respuestas hacia los demás, con irritabilidad; y falta de realización personal en el trabajo (RP) con respuestas negativas hacia sí mismo y el trabajo. En 1982 lo definieron como una «respuesta inadecuada a un estrés emocional crónico cuyos rasgos son agotamiento emocional, despersonalización y un sentimiento de inadecuación a las tareas que ha de realizar», y elaboraron el Maslach Burnout Inventory-Human Services Survey (MBIHSS) dirigido a profesionales de la salud, validado en profesionales mexicanos por Grajales en el año 2001. ${ }^{3}$

El SDP en médicos cirujanos especialistas se considera como un riesgo profesional por la naturaleza de su trabajo. En países europeos, americanos y asiáticos, la prevalencia reporta cifras que oscilan desde el $2.7 \%^{4}$ hasta el $86.5 \%$; en Hispanoamérica su frecuencia es del $14.5 \%$, en América del Sur va del $2.7 \%$ al $86.5 \%$, en Europa es del $7.0 \%$ al $45.0 \%$, en Centroamérica es del 12.0 y en México es del $33.1 \%$ al $42.8 \%$ (Tabla 1).

El conocimiento obtenido ha puesto las bases para realizar intervenciones con el fin de afrontar el estrés laboral crónico, tanto individual como grupal o institucional.

Nuestro objetivo es aportar elementos específicos al estudio del SDP (prevalencia, valores de subescalas y 
Tabla 1. Prevalencia del síndrome de desgaste profesional (burnout) entre médicos cirujanos especialistas, según diversos autores

\begin{tabular}{|c|c|}
\hline Estudio & Resultados \\
\hline Cotrina y Panez (2019) & 2,7\% en Huánuco, Perú \\
\hline Visser, et al. $(2003)^{6}$ & 7,0\% en Holanda \\
\hline Ordenes $(2004)^{13}$ & $9,0 \%$ en Santiago, Chile \\
\hline Girón y Lozano $(2013)^{14}$ & 12,0\% en San Salvador, El Salvador \\
\hline Grau, et al. (2009) ${ }^{16}$ & 14,5\% en Hispanoamérica \\
\hline Escriba, et al. (2008) $)^{15}$ & 22,4\% en España \\
\hline Lugo $(2019)^{20}$ & $24,4 \%$ en Guaria, Venezuela \\
\hline Ferrel, et al. (2010) $)^{17}$ & 28,0\% en Santa Marta, Colombia \\
\hline Goehring, et al. $(2005)^{8}$ & $28,8 \%$ en Suiza \\
\hline Esquivel, et al. (2007) $)^{11}$ & $33,1 \%$ en Distrito Federal, México \\
\hline Miraval $(2017)^{18}$ & 36,7\% en Lima, Perú \\
\hline Castañeda y García de Alba (2019)* & 40,2\% en Guadalajara, México \\
\hline Gutiérrez, et al. (2008) $)^{10}$ & 42,8\% en Pereira, Colombia \\
\hline Borbolla y Domínguez (2007) $)^{12}$ & 42,8\% en Tabasco, México \\
\hline Pejuskovie, et al. (2011) ${ }^{9}$ & $45,0 \%$ en Belgrado, Serbia \\
\hline Zumárraga, et al. (2008)7 & $48,2 \%$ en Quito, Ecuador \\
\hline Castillo y Rosas (2019) & $86,5 \%$ en Guayaquil, Ecuador \\
\hline
\end{tabular}

${ }^{*}$ Resultados del presente estudio.

determinación de factores de riesgo) en especialistas quirúrgicos de tres hospitales regionales de la Seguridad Social, en Guadalajara (Jal.), México.

\section{Método}

Estudio observacional, descriptivo y transversal en 296 especialistas (59 mujeres y 237 varones) para determinar los factores de riesgo y de protección frente al SDP en relación con variables sociodemográficas y laborales. La población de estudio fueron todos los médicos especialistas quirúrgicos adscritos a tres hospitales regionales que cumplían los siguientes requisitos: más de 1 año de antigüedad, estar activo y con disposición para contestar los instrumentos de evaluación. Se excluyó a los médicos becarios, residentes, directivos y demás personal sanitario. Se eliminaron las encuestas incorrectamente cumplimentadas. Se explicaron al personal incluido los motivos y los objetivos del estudio, y se obtuvo su consentimiento informado. Nuestro estudio, por su transversalidad, no distingue apropiadamente causas de efectos.

\section{Instrumentos}

- Cuestionario para el registro de las variables sociodemográficas (sexo, edad, con o sin pareja estable, tiempo con pareja estable, trabaja la pareja y número de hijos) y laborales (área laboral, antigüedad profesional y en el puesto actual de trabajo, turno laboral, tipo de contratación, otro trabajo y horas que le dedica).

- El MBI-HSS, versión traducida y validada para México, con una fiabilidad del 0.57 al 0.80 , que contiene las siguientes subescalas: AE (nueve ítems), DP (cinco ítems) y RP (ocho ítems). Se suman los valores de los ítems para evaluar los niveles de SDP que tiene el trabajador. Como puntos de corte se categorizaron los niveles bajo, moderado y alto: para $\mathrm{AE}, \leq 18,19-26$ y $\geq 27$; y para $D P, \leq 5,6-9$ y $\geq 10$ ). En contrapartida, la RP funciona en sentido opuesto a las anteriores, con unos valores $\leq 33$, $34-39$ y $\geq 40$; la puntuación baja indicaría baja realización, la intermedia mostraría moderada realización, y la alta sería sensación de logro. Se determinó la presencia del SDP con la afectación de una de las subescalas, ya fuera en su nivel medio o alto.

Se realizó análisis estadístico descriptivo obteniendo cifras absolutas, porcentajes, promedio y desviación estándar. Para la asociación se utilizó la correlación de Pearson. Para el análisis inferencial se aplicó la prueba de ji al cuadrado (con o sin corrección de Yates). Se calcularon las odds ratios (OR), con un intervalo de confianza del 95\% (IC 95\%), mediante los paquetes estadísticos SPSS 15.0 y Epi-info V 6.1, considerando significativo un valor de $p \leq 0.05$.

\section{Resultados}

De las 320 encuestas distribuidas, cumplieron con los criterios de estudio y se completaron correctamente 296; las 24 restantes se eliminaron por no reunir los pautas establecidas. Se obtuvo una tasa de respuesta del $92.5 \%$.

En el perfil sociodemográfico destaca la afectación por burnout en el sexo femenino, con el 44.0 vs. el $36 \%$ en los varones $(p<0.05)$, con una edad media de $45.0 \pm 7.0$ años. El grupo de edad menor de 40 años predominó con el $61.0 \%$ ( $p<0.05)$. El $51.0 \%$ $(p<0.05)$ no tenía pareja estable, el tiempo promedio con esta fue de $15.4 \pm 6.0$ años, el $55.0 \%(p<0.05)$ tenía menos de 15 años con ella, en el $37.0 \%$ no 
Tabla 2. Relación de las variables sociodemográficas y laborales con la presencia o ausencia de síndrome de desgaste profesional (burnout) entre los médicos cirujanos especialistas de tres hospitales regionales de la ciudad de Guadalajara (Jal.), México, en $2019(n=296)$

\begin{tabular}{|c|c|c|c|}
\hline \multirow[t]{2}{*}{ Variables analizadas } & \multicolumn{2}{|c|}{ SDP, n (\%) } & \multirow[t]{2}{*}{$\mathrm{p}$} \\
\hline & Sí & No & \\
\hline & $119(40.2)$ & $177(59.8)$ & \\
\hline \multicolumn{4}{|c|}{ Perfil sociodemográfico } \\
\hline \multicolumn{4}{|l|}{ Sexo } \\
\hline Masculino & $93(39.0)$ & $144(61.0)$ & 0.49 \\
\hline Femenino & $26(44.0)$ & $33(56.0)$ & \\
\hline \multicolumn{4}{|l|}{ Grupo de edad } \\
\hline$<40$ años & $47(61.0)$ & $30(39.0)$ & $0.00001^{*}$ \\
\hline > 40 años & $72(33.0)$ & $147(67.0)$ & \\
\hline \multicolumn{4}{|l|}{ Estado civil } \\
\hline Con pareja estable & $70(36.0)$ & $129(64.0)$ & $0.01^{*}$ \\
\hline Sin pareja estable & $49(51.0)$ & $48(49.0)$ & \\
\hline \multicolumn{4}{|c|}{ Tiempo con pareja estable } \\
\hline$<15$ años & $40(55.0)$ & $32(45.0)$ & $0.00000^{*}$ \\
\hline > 15 años & $30(23.0)$ & $97(77.0)$ & \\
\hline \multicolumn{4}{|l|}{ Trabaja la pareja } \\
\hline Sí & $45(34.0)$ & $88(66.0)$ & 0.57 \\
\hline No & $25(37.0)$ & $41(63.0)$ & \\
\hline \multicolumn{4}{|l|}{ Hijos } \\
\hline Sí & $74(36.0)$ & $127(64.0)$ & 0.08 \\
\hline No & $45(48.0)$ & $50(52.0)$ & \\
\hline
\end{tabular}

\begin{tabular}{|c|c|c|c|}
\hline \multicolumn{4}{|c|}{ Perfil laboral } \\
\hline \multicolumn{4}{|l|}{ Área laboral } \\
\hline Traumatología y ortopedia & $29(61.0)$ & $18(39.0)$ & $0.0002^{*}$ \\
\hline Gineco-obstetricia & $29(54.0)$ & $24(46.0)$ & \\
\hline Cirugía general & $20(32.0)$ & $41(68.0)$ & \\
\hline Oftalmología & $12(46.0)$ & $14(54.0)$ & \\
\hline Otorrinolaringología & $10(30.0)$ & $23(70.0)$ & \\
\hline Maxilofacial & $2(25.0)$ & $6(75.0)$ & \\
\hline Anestesiología & $13(20.0)$ & $49(80.0)$ & \\
\hline Neurocirugía & $1(50.0)$ & $1(50.0)$ & \\
\hline Oncología quirúrgica & $3(75.0)$ & $1(25.0)$ & \\
\hline \multicolumn{4}{|l|}{ Antigüedad profesional } \\
\hline$<10$ años & $38(58.0)$ & $27(42.0)$ & $0.0006^{*}$ \\
\hline > 10 años & $81(36.0)$ & $150(64.0)$ & \\
\hline \multicolumn{4}{|l|}{$\begin{array}{l}\text { Antigüedad en el puesto actual } \\
\text { de trabajo }\end{array}$} \\
\hline$<10$ años & $85(51.0)$ & $83(49.0)$ & $0.00002^{*}$ \\
\hline > 10 años & $34(27.0)$ & $94(73.0)$ & \\
\hline Turno laboral & & 9 & \\
\hline Matutino & $69(42.0)$ & $6(58.0)$ & $0.00003^{*}$ \\
\hline Vespertino & $18(22.0)$ & $63(78.0)$ & \\
\hline Nocturno & $19(67.0)$ & $9(33.0)$ & \\
\hline Jornada acumulada & $13(59.0)$ & $9(41.0)$ & \\
\hline \multicolumn{4}{|l|}{ Tipo de contratación } \\
\hline Definitiva & $117(40.0)$ & $171(60.0)$ & 0.37 \\
\hline Temporal & $2(25.0)$ & $6(75.0)$ & \\
\hline
\end{tabular}

Tabla 2. Relación de las variables sociodemográficas y laborales con la presencia o ausencia de síndrome de desgaste profesional (burnout) entre los médicos cirujanos especialistas de tres hospitales regionales de la ciudad de Guadalajara (Jal.), México, en 2019 ( $n=296)$ (Continuación)

\begin{tabular}{|c|c|c|c|}
\hline \multirow[t]{2}{*}{ Variables analizadas } & \multicolumn{2}{|c|}{ SDP, n (\%) } & \multirow[t]{2}{*}{$p$} \\
\hline & Sí & No & \\
\hline & $119(40.2)$ & $177(59.8)$ & \\
\hline \multicolumn{4}{|l|}{ Otro trabajo } \\
\hline Sí & $52(34.0)$ & $101(66.0)$ & $0.02^{*}$ \\
\hline No & $67(46.0)$ & $76(54.0)$ & \\
\hline \multicolumn{4}{|c|}{ Tiempo que labora en el otro } \\
\hline trabajo & $9(30.0)$ & $21(70.0)$ & 0.60 \\
\hline$<4$ horas & $43(35.0)$ & $80(65.0)$ & \\
\hline$>4$ horas & & & \\
\hline
\end{tabular}

trabajaba la pareja y el 48.0\% $(p<0.05)$ manifestaron no tener hijos.

En el perfil laboral destacó la especialidad de oncología quirúrgica con el $75.0 \%(p<0.05)$. La antigüedad profesional tuvo un promedio de $15.4 \pm 6.3$ años, y en el puesto actual de trabajo $10.2 \pm 7.3$ años. El grupo de menos de 10 años de antigüedad laboral fue el $58.0 \%(p<0.05)$ y sobresalió con el $51.0 \%$ el grupo de menos de 10 años de antigüedad en el puesto actual de trabajo. El $67.0 \%$ manifestó laborar en el turno nocturno. El $40.0 \%$ tenía contratación definitiva $(p<0.05)$. El $46.0 \%$ no tenía otro trabajo y el $35.0 \%$ laboraba más de 4 horas en él $(p<0.05)$.

EI SDP se detectó en 119 (40.2\%) participantes, de los cuales $59(49.5 \%)$ tuvieron una subescala afectada, $31(26.0 \%)$ dos y $29(24.5 \%)$ tres.

En lo referente al hospital donde realizan su práctica profesional, la frecuencia del SDP predominó en $46(38.6 \%)$ de ellos en el hospital regional $\mathrm{C}$, seguido del B con $44(36.9 \%)$ y el A con $34(24.5 \%)$.

Posteriormente se caracterizaron dos grupos: uno afectado y otro sin afectar. Inmediatamente se realizó una asociación con cada una de las variables sociodemográficas y laborales. Obsérvese en la tabla 2 que ser mujer, que no trabaje la pareja, no tener hijos, tener contratación definitiva y laborar más de 4 horas en otro trabajo se asociaron como variables de riesgo con la presencia de SDP. Por otra parte, tener menos de 40 años (OR = 3.20; IC 95\%, 1.81 - 5.68), menos de 15 años con pareja estable (OR $=4.04$; IC 95\%, 2.08-7.89), la especialidad de oncología quirúrgica, menos de 10 años de antigüedad profesional (OR = 2.61; IC 95\%, 1.43-4.76) y en el puesto actual 
de trabajo (OR = 2,83; IC 95\%, 1.68-4.79) y laborar en jornada nocturna se asociaron como variables de riesgo con la presencia del SDP y se comportan como factores de riesgo.

Son factores protectores frente al SDP no tener pareja estable $(\mathrm{OR}=0.53$; IC 95\%, $0.31-0.90)$ y no tener otro trabajo (OR = 0.58; IC 95\%, $0.36-0.96)$.

Se asociaron como variables de riesgo con la presencia de SDP en cuanto al AE el ser mujer, no tener pareja estable, que no trabaje esta, no tener hijos, tener contratación definitiva y trabajar menos de 4 horas. Además, tener menos de 40 años (OR = 3.86; IC 95\%, $2.14-6.97$ ), menos de 15 años con pareja estable (OR = 3.61; IC 95\%, 1.77 - 7.39), la especialidad de oncología quirúrgica y tener menos de 10 años de antigüedad profesional $(\mathrm{OR}=2.74$; IC $95 \%, 1.49-5.07)$ y en el puesto actual de trabajo $(\mathrm{OR}=3.41$; IC 95\%, 1.86-6.28) se asociaron como variables de riesgo con la presencia de SDP y se comportan como factores de riesgo.

Se comporta como factor protector contra SDP en presencia de $\mathrm{AE}$ el no tener otro trabajo $(\mathrm{OR}=0.59$; IC 95\%, 0.34 - 0.99).

Se asociaron como variables de riesgo con la presencia de SDP en cuanto a DP ser mujer, no tener hijos, llevar menos de 10 años en el puesto actual de trabajo, laborar en jornada nocturna, tener contratación temporal, no tener otro trabajo y laborar menos de 4 horas. Por otro lado, tener menos de 40 años (OR = 4.24; IC 95\%, 2.18 - 8.25), llevar menos de 15 años con pareja estable (OR = 3.19; IC 95\%, 1.35 - 7.66), la especialidad de gineco-obstetricia y tener menos de 10 años de antigüedad profesional $(\mathrm{OR}=3.18$; IC $95 \%, 1.61-6.29)$ se asociaron como variables de riesgo con la presencia del SDP y se comportan como factores de riesgo.

Se comporta como factor protector frente al SDP en presencia de DP el no tener pareja estable (OR = 0.54; IC 95\%, 0.28 - 0.99).

Se asociaron como variables de riesgo con la presencia de SDP en cuanto a RP tener menos de 40 años, no tener pareja estable, que la pareja trabaje, la especialidad de neurocirugía, tener menos de 10 años de antigüedad profesional y en el puesto actual de trabajo, trabajar en turno nocturno, tener contratación definitiva, no tener otro trabajo y laborar más de 4 horas en este. Además, ser varón ( $\mathrm{OR}=3.20$; IC $95 \%, 1.24$ - 8.71) y llevar menos de 15 años con pareja estable (OR = 2.37; IC 95\%, 1.14 - 4.95) se asociaron como variables de riesgo con la presencia del SDP y se comportan como factor de riesgo.
Es un factor protector contra el SDP en presencia de RP el no tener hijos (OR = 0.22; IC 95\%, $0.12-0.41$ ).

Se efectuó una correlación con el SDP en las tres subescalas, que mostró una asociación inversamente proporcional de AE $(-0.678 ; p=0.0001)$ y DP $(-0.468$; $p=0.0001)$, y directamente proporcional de RP $(0.641$; $p=0.0001$ ).

Los valores medios en las subescalas de AE (14.8), DP (5.1) y RP (39.1) mostraron niveles bajos en las subescalas AE y DP, y nivel alto en RP.

\section{Discusión}

EI SDP crece en complejidad a medida que se profundizan la identificación y la evaluación de posibles causas, determinantes y procesos adyacentes. Nuestro estudio en la totalidad de los médicos cirujanos especialistas de tres hospitales de Guadalajara (Jal.), México, aporta elementos para constatar la importancia y la magnitud del SDP en personal de salud, y sus factores de riesgo y de protección asociados.

Como restricciones del trabajo están su diseño transversal y que el instrumento de recolección de datos, por ser estructurado, disminuye las respuestas de los sujetos de estudio a las opciones empleadas. Otras restricciones son el sesgo de memoria y la reserva individual a que la información otorgada se usara de forma negativa. El primer aspecto se solventó al utilizar cuestionarios validados, y el segundo se trató de evitar mediante el uso de preguntas concretas y objetivas, asegurando el anonimato de los informantes.

Nuestro estudio logró un $92.5 \%$ de respuesta, que garantiza un tamaño representativo de los médicos cirujanos especialistas en estos hospitales y refleja una frecuencia alta respecto a otros estudios reportados en la literatura ${ }^{6-12}$, pero por debajo de otros ${ }^{13}$.

El sexo femenino fue el que presentó mayor prevalencia en nuestro estudio, acorde a la literatura médica revisada ${ }^{5,9,14,15}$, pero no coincidiendo con otros autores que hallan un predominio del sexo masculino1, ${ }^{6-8,10-12,16-20}$. Gutiérrez, et al..$^{10}$ en $200^{9}$ y Ordenes $^{13}$ en 2004 no encontraron diferencias significativas.

En cuanto a la edad, el mayor número de casos se presentó en el grupo de menos de 40 años y se asoció de manera significativa, ajustándose a las investigaciones de Castillo y Rosas ${ }^{5}$ en 2019, Girón y Lozano $^{14}$ en 2013 y Lugo ${ }^{20}$ en 2019, pero no coincidiendo con otros autores ${ }^{3,6-12,16-19}$ que reportaron un predominio de los de mayores de 40 años. Ordenes ${ }^{10}$ no halló diferencias significativas. 
El hecho de no tener pareja estable se mostró con mayor frecuencia en nuestro estudio, coincidiendo con Gutiérrez, et al. ${ }^{10}$ en 2006, Esquivel, et al. ${ }^{11}$ en 2007 y Lugo ${ }^{20}$ en 2019; en la mayoría de los autores prevaleció el tener pareja estable $e^{4-7.9,14,16,20}$. Ordenes ${ }^{10}$ no encontró diferencias significativas.

En nuestra investigación destacó el tener menos de 15 años con pareja estable y se asoció de manera significativa, y además el que la pareja trabajara también destacó. No fueron reportadas estas variables en los artículos examinados.

El no tener hijos sobresalió en nuestro estudio, pero esta variable no fue reportada en los trabajos revisados. No obstante, para Esquivel, et al..$^{11}$ en 2007 y Grau, et al..$^{16}$ en 2009 se destacó el tener hijos y para ellos tuvo un efecto protector.

Los médicos cirujanos especialistas que sobresalieron en el área laboral en nuestro estudio fueron los de oncología quirúrgica (75.0\%) y se asoció significativamente, coincidiendo con Escriba, et al..$^{15}$ en 2008. Para otros autores destacaron anestesiología $a^{6,18,19}$, cirugía general ${ }^{7,13,17,18}$, gineco-obstetricia ${ }^{14}$, traumatología y ortopedia ${ }^{16}$.

Tener una antigüedad profesional menor de 10 años se destacó en nuestra investigación y se asoció significativamente, lo que concuerda con Cotrina y Panez ${ }^{3}$ en 2019 y no coincide con otros autores ${ }^{7,10,12,15-17,}$. Ordenes ${ }^{13}$ no encontró diferencias significativas.

La antigüedad en el puesto actual de trabajo fue menor de 10 años y se manifestó significativamente; esta variable no fue reportada en los estudios revisados.

En lo referente al turno laboral, destacó el nocturno y se asoció significativamente, concordando con Esquivel, et al. ${ }^{11}$ en 2007.

El tipo de contratación definitiva fue el que predominó en nuestro estudio y coincide con la investigación de Cotrina y Panez ${ }^{3}$ en 2019.

No tener otro trabajo destacó en nuestra investigación y se asoció significativamente, y actúa como factor protector. Nuestro hallazgo no coincide con Zumárraga, et al. ${ }^{7}$ en 2008, quienes destacó el hecho de tenerlo.

Laborar más de 4 horas prevaleció en esta investigación, pero no fue manifestado por otros autores.

Para contextualizar nuestro estudio se hizo una amplia revisión sobre la prevalencia del SDP en médicos cirujanos especialistas (Tabla 1), que nos ubica por encima de la mayoría de las investigaciones revisadas $^{3,6,7,11,14-18,20}$, lo que evidencia la amplia variabilidad en la frecuencia del síndrome, situación que a su vez pone de manifiesto el carácter complejo de sus componentes. No obstante, también nos encontramos por debajo de lo reportado por otros autores ${ }^{5,7,9,10,12}$.

Se nota un rasgo de riesgo consistente para SDP, $A E, D P$ y RP juntos: tener menos de 40 años, no tener pareja estable, llevar menos de 15 años con pareja, no tener hijos, tener menos de 10 años de antigüedad profesional y en el puesto actual de trabajo, trabajar en turno nocturno y no tener otro trabajo; para SDP, $A E$ y RP, contratación definitiva; para SDP y AE, ser mujer, que no trabaje la pareja y especialidad oncología quirúrgica; y para SDP y RP, laborar en otro trabajo más de 4 horas. Este perfil plantea que posiblemente la sociabilidad estructural donde se desarrolla el especialista participa en la determinación de ciertos riesgos.

Los valores medios de la subescalas AE y DP se encuentran dentro de los parámetros bajos de la normalidad, y la puntuación de RP se encuentra dentro del nivel alto.

El establecimiento de una correlación negativa con la presencia de SDP entre las subescalas AE y DP, y positiva con la de RP, está en concordancia con lo encontrado por Salanova, et al. ${ }^{21}$ en el año 2000. Este resultado también ha sido confirmado mediante modelos de ecuaciones estructurales (Schaufeli, et al. ${ }^{22}$, en 2002 y Manzano en $2002^{23}$ ). Se confirma que el AE es la subescala más fiable del síndrome. Lee y Ashforth en $1996^{24}$, y Schaufeli y Enzmann en $1998^{25}$, mencionan que las dos dimensiones centrales del síndrome son el AE y la DP.

\section{Conclusiones}

El SDP es frecuente (40.2\%) entre los médicos cirujanos especialistas. Sus principales factores de riesgo son ser mujer, menor de 40 años de edad, no tener pareja estable, llevar menos de 15 años con pareja y que la pareja no trabaje, no tener hijos, la especialidad de oncología quirúrgica, tener menos de 10 años de antigüedad profesional y en el puesto actual de trabajo, laborar en el turno nocturno, tener contratación definitiva, no tener otro trabajo y laborar más de 4 horas en él. La afectación del AE y la DP se comportan como el síndrome. Los niveles medios de las subescalas se encuentran en general bajos. Hay una correlación negativa entre $A E$ y $D P$, y positiva con la RP con presencia del SDP. Lo anterior nos lleva a considerar la necesidad de establecer medidas preventivas o de intervención de ámbito individual, social u organizativo para reducir la prevalencia encontrada. 


\section{Conflicto de intereses}

Los autores declaran que no existen conflictos de intereses en relación con esta investigación.

\section{Financiamiento}

Los autores declaran que no se recibió ninguna financiación.

\section{Responsabilidades éticas}

Protección de personas y animales. Los autores declaran que los procedimientos seguidos se conformaron a las normas éticas del comité de experimentación humana responsable y de acuerdo con la Asociación Médica Mundial y la Declaración de Helsinki.

Confidencialidad de los datos. Los autores declaran que han seguido los protocolos de su centro de trabajo sobre la publicación de datos.

Derecho a la privacidad y consentimiento informado. Los autores han obtenido el consentimiento informado de los pacientes y/o sujetos referidos en el artículo. Este documento obra en poder del autor de correspondencia.

\section{Bibliografía}

1. Freudenberger M. Staff Burn-out. J Soc Issues. 1974:30:159-66.

2. Maslach C, Jackson S. Maslach Burnout Inventory. Manual. Palo Alto, California: Consulting Psychologist Press, 1981.

3. Grajales G. Estudio de la validez factorial del Maslach Burnout Inventory versión Española en una población de profesionales mexicanos. Línea de Investigación: Instrumentos de medición SELA. 2001:1-10.

4. Cotrina $Y, P a n e z ~ L$. Síndrome de burnout en médicos de tres hospitales de Huánuco. Rev Peru Investig Salud. 2019;3:127-32.

5. Castillo G, Rosas L. Identificación del síndrome de burnout en el personal médico de emergencias de un hospital de segundo nivel. (Tesis), Universidad Espíritu Santo, Guayaquil-Ecuador; 2019.
6. Visser M, Smets E, Oort F, De Haes H. Stress, satisfaction and burnout among Dutch medical specialist. CMAJ. 2003;168:271-5.

7. Zumárraga R, García M, Yépez L. Prevalencia del síndrome de burnout en médicos de tres hospitales públicos de la ciudad de Quito. Rev Fac Cien Med (Quito). 2008;33:96-100.

8. Goehring C, Bouvier M, Künzi B, Bovier P. Psychosocial and professional characteristics of burnout in Swiss primary care practitioners: a cross-sectional survey. Swiss Med Wkly. 2005;135:101-8.

9. Pejuskovie B, Leeié D, Priebe S, Toskovie O. Burnout syndrome among physicians - the role of personality dimensions and coping strategies. Psychiatria Danubina. 2011;23:389-95.

10. Gutiérrez J, Peña J, Montenegro J, Osorio D, Caicedo C, Gallego Y. Prevalencia y factores asociados a "burnout" en médicos especialistas docentes de la Universidad Tecnológica de Pereira. 2006. Rev Med Risaralda. 2008:14:15-22.

11. Esquivel C, Buendía F, Martínez O, Martínez J, Martínez V, Velasco V. Síndrome de agotamiento profesional en personal médicos de un hospital de tercer nivel. Rev Med Inst Mex Seguro Soc. 2007;45:427-36.

12. Borbolla M, Domínguez M. Síndrome de burnout en personal médico familiar y no familiar del Instituto Mexicano del Seguro Social en Tabasco. Salud en Tabasco. 2007;13:577-83.

13. Ordenes N. Prevalencia de burnout en trabajadores del Hospital Roberto del Río. Rev Chil Pediatr. 2004;75:449-54.

14. Girón A, Lozano D. Síndrome de desgaste laboral y sus efectos en el ambiente laboral del personal de salud del Hospital Nacional San Rafael. (Tesis), Universidad Dr. José Matías Delgado. San Salvador-El Salvador; 2013.

15. Escriba V, Artazcoz L, Pérez S. Efecto del ambiente psicosocial y de la satisfacción laboral en el síndrome de burnout en médicos especialistas. Gac Sanit. 2008:22:300-8.

16. Grau A, Flichtentrei D, Suñer R, Prats M, Braga F. Influencia de factores profesionales y transnacionales en el síndrome de burnout en personal sanitario hispanoamericano y español (2007). Rev Esp Salud Pública. 2009;83:215-30.

17. Ferrel R, Sierra E, Rodríguez M. Síndrome de desgaste profesional (burnout) en médicos especialistas de un hospital universitario de la ciudad de Santa Marta, Colombia. Duazary. 2010;7:29-40.

18. Miraval E. Síndrome de burnout en personal médico del Hospital III Emergencias Grau en el período octubre a diciembre del 2016. (Tesis), Universidad Ricardo Palma, Lima-Perú; 2017.

19. Association of Professors of Medicine (APS). Predicting and preventing physician burnout: results from the United States and the Netherlands. Am J Med. 2001;111:170-5.

20. Lugo J. Síndrome de burnout. Factores asociados en médicos de un hospital tipo III. Rev Dig Postgrado. 2019;8:e158.

21. Salanova M, Schaufeli W, Llorens S, Peiró J, Grau R. Desde el "burnout" al "Engagement" ¿Una nueva perspectiva? Revista de Psicología del Trabajo y de las Organizaciones. 2000:16:117-34

22. Schaufeli W, Martínez I, Marqués A, Salanova M, Bakker A. Burnout and engagement in university students: A cross-national study. Journal of Cross-Cultural Psychology. 2002;33:464-81.

23. Manzano G. Bienestar subjetivo de los cuidadores formales de Alzheimer: relaciones con el burnout, engagement y estrategias de afrontamiento. Ansiedad y Estrés. 2002;8:225-44.

24. Lee R, Ashforth B. A meta-analytic examination of the correlates of the three dimensions of job burnout. Journal of Applied Psychology. 1996;81(2):123-33.

25. Schaufeli W, Enzmann D. The burnout companion to study and research: A critical analysis. London. Taylor and Francis, 1998. 